\title{
A SMOOTHNESS CRITERION FOR COMPLEX SPACES IN TERMS OF DIFFERENTIAL FORMS
}

\author{
HÅKAN SAMUELSSON KALM \& MARTIN SERA
}

\begin{abstract}
For a reduced pure dimensional complex space $X$, we show that if Barlet's recently introduced sheaf $\alpha_{X}^{1}$ of holomorphic 1-forms or the sheaf of germs of weakly holomorphic 1-forms is locally free, then $X$ is smooth. Moreover, we discuss the connection to Barlet's well-known sheaf $\omega_{X}^{1}$.
\end{abstract}

\section{Introduction}

Let $X$ be a reduced pure dimensional complex space. In presence of singularities, there exist several natural concepts of holomorphic differential forms on $X$. Beside of the well-known sheaf $\Omega_{X}^{1}$ of Kähler differentials, the sheaf $\Omega_{X}^{1} /$ torsion of strongly holomorphic forms, and the so-called Barlet sheaf $\omega_{X}^{1}$, we will consider the following two in this note. We say that a holomorphic 1 -form $\varphi$ on $X_{\text {reg }}$ is weakly holomorphic if there is a resolution of singularities $\pi: \tilde{X} \rightarrow X$, with $\tilde{X}$ smooth, such that $\pi^{*} \varphi$ extends holomorphically across the exceptional set. By [Gri76, Section II], $\varphi$ is weakly holomorphic if and only if for any holomorphic curve $C \subset X$ not contained in $X_{\text {sing }}$, $\varphi \wedge \bar{\varphi}$ is integrable on the regular part of any small enough open subset in $C$. We will denote the sheaf of germs of weakly holomorphic 1 -forms by $\tilde{\Omega}_{X}^{1}$; thus $\tilde{\Omega}_{X}^{1}=\pi_{*} \Omega_{\tilde{X}}^{1}$. In [Bar18, Section 3], Barlet introduces the coherent analytic subsheaf $\alpha_{X}^{1}$ of $\tilde{\Omega}_{X}^{1}$ which is defined as follows. Using [Ros68, Theorem 3.5] (cf. also [Rie71, § 2], [Bar18, Prop. 2.1.1]), we may assume that $\pi$ is chosen such that $\pi^{*} \Omega_{X}^{1} /$ torsion is locally free. On a small enough open set, let $\varphi_{1}, \ldots, \varphi_{N}$ be holomorphic 1-forms generating $\Omega_{X}^{1}$. Then, $\pi^{* *} \Omega_{X}^{1}$ is the $\mathscr{O}_{\tilde{X}}$-module sheaf on $\tilde{X}$ generated by $\pi^{*} \varphi_{1}, \ldots, \pi^{*} \varphi_{N}$ modulo torsion, where $\pi^{*} \varphi_{k}$ denotes the pullback as differential form. $\alpha_{X}^{1}$ is defined as the direct image sheaf $\pi_{*}\left(\pi^{* *} \Omega_{X}^{1}\right)$. We have $\Omega_{X}^{1}$ /torsion $\subset \alpha_{X}^{1} \subset \tilde{\Omega}_{X}^{1}$ which are proper inclusions in general. Furthermore, there is a pullback functor for $\alpha_{X}^{1}$ (compatible with the pullback of strongly holomorphic forms) which cannot exist for weakly holomorphic forms. The purpose of this note is to show

Theorem 1.1. (i) $X$ is smooth if and only if $\tilde{\Omega}_{X}^{1}$ is a locally free $\mathscr{O}_{X}$-module. (ii) $X$ is smooth if and only if $\alpha_{X}^{1}$ is a locally free $\mathscr{O}_{X}$-module.

Date: May 18, 2020.

2010 Mathematics Subject Classification. 32C35 (32C15).

The last author was supported by the German Research Foundation (DFG, grant SE 2677/1) and the Knut and Alice Wallenberg Foundation. 
The only if-parts are of course trivial. Our contribution is essentially the observation that if $\tilde{\Omega}_{X}^{1}$ or $\alpha_{X}^{1}$ is locally free, then $X$ is normal and the tangent sheaf, $\mathscr{T}_{X}$, is locally free. That $X$ then is smooth follows by an argument of van Straten and Steenbrink, vSS85, Section 1.6], elaborated by Greb, Kebekus, Kovács, and Peternell, GKKP, Theorem 6.1] which we outline for completeness (see Proposition 2.2). The (i)-part was already proven by Kersken with a different approach in Ker88, Satz 3.1].

Obviously, Theorem 1.1 generalizes the classical smoothness criterion: if the sheaf of Kähler differentials $\Omega_{X}^{1}$ or the strongly holomorphic 1-forms $\Omega_{X}^{1}$ /torsion is locally free, then $X$ is smooth. It is a well-known problem whether there is a smoothness criterion in terms of the sheaf $\omega_{X}^{1}$ introduced by Barlet, [Bar78, Section 1]. $\omega_{X}^{1}$ may be defined as the sheaf of $\bar{\partial}$-closed currents on $X$ modulo the $\bar{\partial}$-closed currents with support in the singular set $X_{\text {sing, }}$ cf. [Bar78, Prop. 4]. Sections of $\omega_{X}^{1}$ over $U \backslash A$, where $U \subset X$ is open and $A \subset U$ is an analytic set with $\operatorname{codim}_{U} A \geq 2$, extend across $A$ and, moreover, $\omega_{X}^{1}$ is always torsion free, see [Bar78, Section 1]. It follows that $\omega_{X}^{1}$ is reflexive if $X$ is normal, see [Bar78, Section 2]. In view of Theorem 1.1, a smoothness criterion in terms of $\omega_{X}^{1}$ follows for spaces $X$ such that $\tilde{\Omega}_{X}^{1} \simeq \omega_{X}^{1}$. In general, $\tilde{\Omega}_{X}^{1} \subsetneq \omega_{X}^{1}$ and the quotient $\omega_{X}^{1} / \tilde{\Omega}_{X}^{1}$ is related to the $s^{(1)}$-invariant of isolated singularities, see [Yau82]. However, Flenner's main result in [Fle88] implies that $\tilde{\Omega}_{X}^{1}$ is reflexive if $X$ is normal and $\operatorname{codim}_{X} X_{\text {sing }} \geq 3$. If $X$ has klt singularities, then $\tilde{\Omega}_{X}^{1}$ is reflexive as well, see [GKKP, Theorem 1.4]. In both cases, we obtain that $\tilde{\Omega}_{X}^{1}=\omega_{X}^{1}$ since on a normal space a reflexive sheaf is uniquely determined by its restriction to the regular part. Moreover, Pinkham and Wahl have shown that this equality also holds if $X$ is a surface with rational singularities, see, see [Pin80, Appendix] (cf. also Wah75, $\S 4-5]$ ). We remark that this last result does not hold in general in positive characteristic. Inspired by Pinkham, Pin80, Prop. 1, Appendix], we get a sufficient cohomological condition ensuring the equality $\tilde{\Omega}_{X}^{1}=\omega_{X}^{1}$, see Proposition 2.3 below, and we conclude the following corollary of Theorem 1.1 .

Corollary 1.2. Let $X$ be a reduced Stein space and $M \rightarrow X$ a resolution of singularities with exceptional divisor $E$ having normal crossings. If $\omega_{X}^{1}$ is locally free and $H_{E}^{1}\left(\Omega_{M}^{1}(\log E) \otimes \mathscr{O}(-E)\right)=0$, then $X$ is smooth.

In Corollary 2.5 below, we collect all cases discussed in this note where the local freeness of $\omega_{X}^{1}$ implies $X$ is smooth.

Let us put this in the context of the Lipman-Zariski conjecture, Lip65, Introduction], which states that $X$ is smooth if and only if $\mathscr{T}_{X}$ is locally free. Lipman Lip65, Theorem 1] showed that if $\mathscr{T}_{X}$ is locally free, then $X$ is at least normal. The conjecture has been proved assuming a priori, e.g., that $\operatorname{codim}_{X} X_{\text {sing }} \geq 3$ or that $X$ is a klt space, see [vSS85, Section 1.6], [Fle88, Corollary], [GKKP, Theorem 
6.1], and the references therein. On a normal space the Lipman-Zariski conjecture may be reformulated in terms of $\omega_{X}^{1}$. Since the dual sheaf $\mathscr{T}_{X}^{*}$ and $\omega_{X}^{1}$ are reflexive, $\mathscr{T}_{X}^{*} \simeq \omega_{X}^{1}$ if $X$ is normal. Therefore, since $\mathscr{T}_{X}$ is reflexive (as the dual of the Kähler differentials), on a normal space $X$, the Lipman-Zariski conjecture is equivalent to the statement that $X$ is smooth if and only if $\omega_{X}^{1}$ is locally free. We conclude that the Lipman-Zariski conjecture holds for complex spaces satisfying the cohomology condition in Corollary 1.2, in particular, for surfaces with rational singularities (the latter was proved also by Kersken, see [Ker88, (3.3)]).

Acknowledgment: We would like to thank Jan Stevens for fruitful discussions and suggestions and the anonymous referee for valuable comments which helped to improve the paper.

\section{Proofs}

A crucial ingredient of the proof of Theorem 1.1 is the following proposition which generalizes a result by the second author in [Ser16, Sect. 4]:

Proposition 2.1. If $\mathscr{E}$ is a locally free sheaf of positive rank on a reduced complex space $X$ such that there exist a proper modification $\pi: Z \rightarrow X$ with $Z$ normal and a coherent analytic sheaf $\mathscr{F}$ with $\pi_{*} \mathscr{F} \simeq \mathscr{E}$, then $X$ is normal.

Proof. We only need to prove that $X$ is locally irreducible. That $X$ is normal then follows from [Ser16, Theorem 4.6].

Let $x \in X_{\text {sing }}$ and assume to get a contradiction that $X$ is reducible at $x$. Then, there is a connected neighbourhood $U$ of $x$ and a decomposition of $U$ in irreducible components $U_{1}, \ldots, U_{m}, m \geq 2$. Since $Z$ is normal, the preimages $V_{i}=\pi^{-1}\left(U_{i}\right)$, $i=1, \ldots, m$ are pairwise disjoint. Set $V:=\pi^{-1}(U)$. We may assume that $\mathscr{O}_{U}^{r} \simeq \mathscr{E}_{U} \simeq$ $\pi_{*} \mathscr{F}_{V}$. Let $\Phi: \mathscr{O}_{U}^{r} \rightarrow \pi_{*} \mathscr{F}_{V}$ be the composition of these isomorphisms. Consider $e_{1}:=\left(\begin{array}{llll}1 & 0 & \ldots & 0\end{array}\right)^{T} \in \mathscr{O}^{r}(U)$ and $g:=\Phi\left(e_{1}\right) \in \pi_{*} \mathscr{F}(U)=\mathscr{F}(V)$. We denote the restriction of $g$ to $V_{i}$ by $\tilde{g}_{i} \in \mathscr{F}\left(V_{i}\right)$. Let $g_{i} \in \mathscr{F}(V)$ be the trivial extension of $\tilde{g}_{i}$, which is well defined since all $V_{i}$ and $V \backslash V_{i}$ are disjoint, open and closed. Then, $g=\sum g_{i}$ and, in particular, $e_{1}=\sum \Phi^{-1}\left(g_{i}\right)$. We obtain that $\Phi^{-1}\left(g_{1}\right)$ is $e_{1}$ on $U_{1} \backslash X_{\text {sing }}$ and 0 on the other irreducible components. This contradicts the fact that $\Phi^{-1}\left(g_{1}\right) \in \mathscr{O}^{r}(U)$ is a holomorphic function on the connected $U$ completing the proof.

When $X$ is normal, a coherent analytic sheaf $\mathscr{S}$ is reflexive if and only if $\mathscr{S}$ is torsion-free and sections of $\mathscr{S}$ extend across analytic sets of codimension at least 2. In particular, every section of a reflexive sheaf on the regular part of $X$ extends across the singular set. 
Proposition 2.2. Let $X$ be a normal complex space, and $\mathscr{E}$ be a coherent analytic subsheaf of $\tilde{\Omega}_{X}^{1}$ such that $\mathscr{E}_{X_{\mathrm{reg}}} \simeq \Omega_{X_{\mathrm{reg}}}^{1}$. If $\mathscr{E}$ is locally free, then $X$ is smooth.

Proof. We get $\mathscr{T}_{X_{\text {reg }}} \simeq\left(\tilde{\Omega}_{X_{\text {reg }}}^{1}\right)^{*} \simeq \mathscr{E}_{X_{\text {reg }}^{*}}$. Since $\mathscr{T}_{X}$ is reflexive, this isomorphism extends across the singular set and so, $\mathscr{T}_{X} \simeq \mathscr{E}^{*}$ is locally free.

With $\mathscr{T}_{X}$ and $\mathscr{E}$ locally free and $\mathscr{E} \subset \tilde{\Omega}_{X}^{1}$, we obtain the smoothness of $X$ in the same way as in the proof of Theorem 6.1 in [GKKP]:

To get a contradiction we assume that $X$ is not smooth. Let $x \in X$ be a singular point of $X$. By shrinking $X$ to a small neighbourhood of $x$, we may assume that $\mathscr{T}_{X}$ and $\mathscr{E}$ are free. There exists a resolution of singularities, $\rho_{X}: R(X) \rightarrow X$ with simple normal crossing exceptional divisor. We may assume that $\rho$ is functorial with respect to smooth morphisms (flat submersions), see, e. g., Theorem 3.45 in [Kol07]. The functorial property means that if $f: X \rightarrow Y$ is a smooth morphism, then we can lift $f$ to the resolutions, i. e., there exists a smooth morphism $R(f): R(X) \rightarrow R(Y)$ such that $f \circ \rho_{X}=\rho_{Y} \circ R(f)$. Let $E$ denote the exceptional divisor of $\rho:=\rho_{X}$ in $R:=R(X)$ which is not empty (since $X$ is not smooth). Since $\mathscr{T}_{X}$ is free, there is a frame of sections $\theta_{1}, \ldots, \theta_{n}$ which generates $\mathscr{T}_{X}$ on $X$. Since the singular set of $X$ is invariant under any (local) automorphism and $\rho$ is functorial, Corollary 4.7 in GKK] gives us

$$
\mathscr{T}_{X} \simeq \rho_{*} \mathscr{T}_{R}(-\log E)
$$

where $\mathscr{T}_{R}(-\log E)$ denotes the logarithmic tangent sheaf (i. e., vector fields which are tangent to $E$ in smooth points of $E ; \mathscr{T}_{R}(-\log E)$ is the dual of the logarithmic differential forms $\Omega_{R}^{1}(\log E)$ ). Hence, (we are in the special situation that) we may lift the vector fields $\theta_{i}$ to logarithmic vector fields

$$
\tilde{\theta}_{i} \in H^{0}\left(R, \mathscr{T}_{R}(-\log E)\right) \subset H^{0}\left(R, \mathscr{T}_{R}\right) .
$$

Since $\mathscr{E} \simeq \mathscr{T}_{X}^{*}$, there is a dual frame $\omega_{1}, \ldots, \omega_{n} \in \mathscr{E} \subset \tilde{\Omega}_{X}^{1}$ of $\theta_{1}, \ldots, \theta_{n}$. Since $\tilde{\Omega}_{X}^{1}=$ $\rho_{*} \Omega_{R}^{1}, \omega_{i}$ is given by holomorphic forms $\tilde{\omega}_{1}, \ldots, \tilde{\omega}_{n} \in H^{0}\left(R, \Omega_{R}^{1}\right)$ on $R$. On $R \backslash E$, we have $\tilde{\omega}_{i}\left(\tilde{\theta}_{j}\right)=\omega_{i}\left(\theta_{j}\right)=\delta_{i j}$. These equalities extend to $E$ by continuity. In particular, $\tilde{\theta}_{1}(p), \ldots, \tilde{\theta}_{n}(p)$ are linear independent for every point $p \in E$. We obtain a contradiction since all $\tilde{\theta}_{i}(p)$ are in $T_{p} E$ (because of (2.1) ) with $\operatorname{dim} T_{p} E=n-1$ for $p \in E_{\text {reg }}$ This completes the proof.

Proof of Theorem 1.1. As already mentioned, we of course only need to prove that local freeness of $\tilde{\Omega}_{X}$ or $\alpha_{X}^{1}$ implies $X$ is smooth: Since $\tilde{\Omega}_{X}^{1}:=\pi_{*} \Omega_{M}^{1}$ and $\alpha:=$ $\pi_{*}\left(\pi^{* *} \Omega_{X}^{1}\right)$, the assumption implies $X$ is normal by Proposition 2.1. The smoothness of $X$ follows now by Proposition 2.2 .

\footnotetext{
${ }^{1}$ For pure dimensional complex spaces $X$ and $Y$, a holomorphic morphism $f: X \rightarrow Y$ is a submersion if the relative $\Omega_{X \mid Y}^{1}$ is locally free of $\operatorname{rank} \operatorname{dim} X-\operatorname{dim} Y$ (which is the case if and only if $f$ can be seen locally as a projection).
} 
Corollary 1.2 follows from Theorem 1.1 and the following proposition which is a generalization of a result of Pinkham (see [Pin80, Proposition 1, Appendix]).

Proposition 2.3. Let $X$ be a reduced Stein space, $\pi: M \rightarrow X$ a resolution of singularities with $E$ as exceptional divisor having normal crossings. If

$$
H_{E}^{1}\left(\Omega_{M}^{1}(\log E) \otimes \mathscr{O}(-E)\right)=0
$$

then

$$
\tilde{\Omega}_{X}^{1}=\omega_{X}^{1}
$$

Thereby, $\Omega_{M}^{1}(\log E)$ denotes the logarithmic differential forms. Pinkham proved that

$$
H_{E}^{1}\left(\Omega_{M}(\log E)\right)=0=H^{0}\left(M, \Omega_{M}(\log E) \otimes \mathscr{O}_{E}\right)
$$

implies (2.2). Additionally, J. Wahl proved that if $X$ is a surface with rational singularities, then (2.2) is satisfied, too (see [Pin80, ThÃl'orẤlme (Wahl)], using Wah75, §, 4-5]).

The condition (2.2) does not imply that $\alpha_{X}^{1} \simeq \omega_{X}^{1}$ : By [Bar18, Section 6.2], we have

$$
\Omega_{S_{k}}^{1} / \text { torsion }=\alpha_{S_{k}}^{1} \subsetneq \tilde{\Omega}_{S_{k}}^{1}=\omega_{S_{k}}^{1}
$$

for the surface $S_{k}:=\left\{(x, y, z): x y=z^{k}\right\}$ in $\mathbb{C}^{3}$. Yet, $S_{k}$ has rational singularities so that (2.2) is satisfied for a resolution of singularities of $S_{k}$ (see [Pin80, ThÃl'or Âlme (Wahl)]).

Proof of Proposition [2.3. To shorten the notation, let us define $\Omega_{M}^{1}(-E+\log E):=$ $\Omega_{M}^{1}(\log E) \otimes \mathscr{O}(-E)$.

As first step, we will prove that the inclusion $H^{0}\left(M, \Omega_{M}^{1}\right) \hookrightarrow H^{0}\left(M^{*}, \Omega_{M}^{1}\right)$ induced by the restriction $\cdot_{M^{*}}$ is surjective with $M^{*}:=M \backslash E$ : The short exact sequence

$$
\left.0 \rightarrow \Omega_{M}^{1}(-E+\log E)\right|_{E} \rightarrow \Omega_{M}^{1}(-E+\log E) \rightarrow \Omega_{M^{*}}^{1}(-E+\log E) \rightarrow 0
$$

gives us a long exact sequence of cohomology whose first $H^{1}$-term vanishes by our assumption (2.2). Hence, the restriction map

$$
H^{0}\left(M, \Omega_{M}^{1}(-E+\log E)\right) \rightarrow H^{0}\left(M^{*}, \Omega_{M}^{1}(-E+\log E)\right)
$$

is surjective. Since it is already an inclusion of sets, we get

$$
H^{0}\left(M, \Omega_{M}^{1}(-E+\log E)\right)=H^{0}\left(M^{*}, \Omega_{M}^{1}(-E+\log E)\right)=H^{0}\left(M^{*}, \Omega_{M}^{1}\right) .
$$

Let us consider a point $p$ in $M$ and coordinates $x_{1}, \ldots, x_{n}$ of $M$ on a neighbourhood of $p$ such that $E=\left\{x_{1} \cdot \ldots \cdot x_{\tau}=0\right\}$. Then $d x_{1} / x_{1}, \ldots, d x_{\tau} / x_{\tau}$ and $d x_{\tau+1}, \ldots, d x_{n}$ give us a basis of $\Omega_{M}^{1}(\log E)_{p}$. Furthermore, $\left\{x_{1} \cdot \ldots \hat{x}_{i} . . \cdot x_{\tau} d x_{i}\right\}_{i=1}^{\tau} \cup\left\{x_{1} \cdot \ldots \cdot x_{\tau} d x_{j}\right\}_{j=\tau+1}^{n}$ is a basis for $\Omega_{M}^{1}(-E+\log E)_{p}$, in particular $\Omega_{M}^{1}(-E+\log E)_{p} \subset \Omega_{M, p}^{1}$. We obtain the 
following chain of inclusions, which then have to be equalities:

$$
H^{0}\left(M^{*}, \Omega_{M}^{1}\right) \stackrel{(2.4)}{=} H^{0}\left(M, \Omega_{M}^{1}(-E+\log E)\right) \subset H^{0}\left(M, \Omega_{M}^{1}\right) \subset H^{0}\left(M^{*}, \Omega_{M}^{1}\right) .
$$

As second step, we want to use the global extension property proven in the first step to obtain the local extension: Since $\omega_{X}^{1}$ is coherent and $X$ is Stein, Cartan's Theorem A implies that there exists an epimorphism of sheaves

$$
\mathscr{O}_{X}^{N} \rightarrow \omega_{X}^{1},\left(f_{i, p}\right)_{i=1}^{N} \mapsto \sum_{i} f_{i, p} \cdot \varphi_{i, p}
$$

(on the whole $X$ ) given by sections $\varphi_{1}, \ldots, \varphi_{N} \in \omega_{X}^{1}(X) \subset H^{0}\left(M^{*}, \Omega_{M}^{1}\right)$. In the first step, we proved that $\varphi_{i}$ can be extended to sections in $H^{0}\left(M, \Omega_{M}^{1}\right)=H^{0}\left(X, \tilde{\Omega}_{X}^{1}\right)$. If $\eta_{p}$ is a germ in $\omega_{X, p}^{1}$, then there exist holomorphic germs $f_{1, p}, \ldots, f_{N, p} \in \mathscr{O}_{X, p}$ such that $\eta_{p}=\sum_{i=1}^{p} f_{i, p} \varphi_{i, p}$. Since $\varphi_{i, p} \in \tilde{\Omega}_{X, p}^{1}$, we get that $\eta_{p}$ can be considered as a germ of $\tilde{\Omega}_{X, p}^{1}$ as claimed.

If $\operatorname{codim} X_{\text {sing }}$ is at least 2, then $\omega_{X}^{1}=i_{*} \Omega_{X_{\text {reg }}}^{1}$ [Bar78, end of Section 2] (where $\left.i: X_{\text {reg }} \hookrightarrow X\right)$. This is not so for codim $X_{\text {sing }}=1$ in general. However, we obtain the following corollary of Proposition 2.3 by slight modifications of the proof.

Corollary 2.4. Let $X$ be a reduced complex space, $\pi: M \rightarrow X$ a resolution of singularities with $E$ as exceptional divisor having normal crossings.

(a) If $X$ is Stein, $i_{*} \Omega_{X_{\mathrm{reg}}}^{1}$ is coherent, and

$$
H_{E}^{1}\left(\Omega_{M}^{1}(\log E) \otimes \mathscr{O}(-E)\right)=0, \quad \text { or }
$$

(b) if for all small enough open sets $U \subset X$,

$$
H_{E}^{1}\left(\Omega_{\pi^{-1}(U)}^{1}(\log E) \otimes \mathscr{O}(-E)\right)=0,
$$

then

$$
\tilde{\Omega}_{X}^{1}=\omega_{X}^{1}=i_{*} \Omega_{X_{\text {reg }}}^{1}
$$

Proof. We follow the same arguments as in the proof of Proposition 2.3. In case the conditions of (a) are satisfied, we obtain in a first step the global extension property:

$$
H^{0}\left(X, \tilde{\Omega}_{X}^{1}\right)=H^{0}\left(M, \Omega_{M}^{1}\right)=H^{0}\left(M^{*}, \Omega_{M}^{1}\right)=H^{0}\left(X_{\mathrm{reg}}, \Omega_{X_{\mathrm{reg}}}^{1}\right) .
$$

Replacing $\omega_{X}^{1}$ by $i_{*} \Omega_{X_{\mathrm{reg}}}^{1}$ in the second step of the proof above, we get the local extension property (2.5).

Only using the first step of the proof of Proposition 2.3, we obtain that the condition (b) implies that the inclusion $H^{0}\left(\pi^{-1}(U), \Omega_{\pi^{-1}(U)}^{1}\right) \hookrightarrow H^{0}\left(\pi^{-1}(U) \backslash E, \Omega_{\pi^{-1}(U)}^{1}\right)$ is surjective for all small enough open sets $U \subset X$, i. e., $\tilde{\Omega}_{X}^{1}(U)=i_{*} \Omega_{X_{\text {reg }}}^{1}(U)$. This implies that the inclusions $\tilde{\Omega}_{X}^{1} \subset \omega_{X}^{1} \subset i_{*} \Omega_{X_{\text {reg }}}^{1}$ are equalities as claimed. 
We conclude this work with collecting cases where local freeness of $\omega_{X}^{1}$ implies smoothness of $X$. We get the following corollary of Theorem 1.1 and the above mentioned results.

Corollary 2.5. Let $X$ be a reduced complex space such that one of the following conditions is satisfied:

(i) $X$ is normal and $\operatorname{codim} X_{\text {sing }} \geq 3$.

(ii) $X$ is normal and has klt singularities.

(iii) $X$ is a surface with rational singularities, or more general

(iv) $X$ is Stein and satisfies (2.3), or more general

(v) $X$ satisfies the assumptions in Corollary 2.3, or

(vi) $X$ satisfies the conditions (a) or (b) of Corollary 2.4.

Then $X$ is smooth if $\omega_{X}^{1}$ is locally free (or $\mathscr{T}_{X}$ is locally free).

\section{References}

[Bar78] Daniel Barlet, Le faisceau $\omega_{X}$ sur un espace analytique $X$ de dimension pure, Fonctions de plusieurs variables complexes, III (Sém. François Norguet, 1975-1977), Lecture Notes in Math., vol. 670, Springer, Berlin, 1978, pp. 187-204.

[Bar18] _ The sheaf $\alpha_{X}$, Journal of Singularities 18 (2018), 50-83.

[Fle88] Hubert Flenner, Extendability of differential forms on nonisolated singularities, Invent. Math. 94 (1988), no. 2, 317-326.

[GKK] Daniel Greb, Stefan Kebekus, and Sándor J. Kovács, Extension theorems for differential forms and Bogomolov-Sommese vanishing on log canonical varieties, Compos. Math. 146 (2010), no. 1, 193-219.

[GKKP] Daniel Greb, Stefan Kebekus, Sándor J. Kovács, and Thomas Peternell, Differential forms on log canonical spaces, Publ. Math. Inst. Hautes Études Sci. (2011), no. 114, 87-169.

[Gri76] Phillip A. Griffiths, Variations on a theorem of Abel, Invent. Math. 35 (1976), 321-390.

[Ker88] Masumi Kersken, Ein Regularitätskriterium für analytische Algebren, Arch. Math. (Basel) 51 (1988), no. 5, 434-439.

[Kol07] János Kollár, Lectures on resolution of singularities, Annals of Mathematics Studies, vol. 166, Princeton University Press, Princeton, NJ, 2007.

[Lip65] Joseph Lipman, Free derivation modules on algebraic varieties, Amer. J. Math. 87 (1965), 874-898.

[Pin80] Henry Pinkham, Singularités rationnelles de surfaces, Séminaire sur les singularités des surfaces 1976-1977, Lecture Notes in Math., vol. 777, Springer, Berlin, 1980, pp. 147-178.

[Rie71] Oswald Riemenschneider, Characterizing Moišezon Spaces by Almost Positive Coherent Analytic Sheaves, Math. Z. 123 (1971), 263-284.

[Ros68] Hugo Rossi, Picard variety of an isolated singular point, Rice Univ. Studies 54 (1968), no. $4,63-73$.

[Ser16] Martin Sera, A Generalization of Takegoshi's Relative Vanishing Theorem, J. Geom. Anal. 26 (2016), no. 3, 1891-1912. 
[vSS85] Duco van Straten and Joseph Steenbrink, Extendability of holomorphic differential forms near isolated hypersurface singularities, Abh. Math. Sem. Univ. Hamburg 55 (1985), 97110.

[Wah75] Jonathan M. Wahl, Vanishing theorems for resolutions of surface singularities, Invent. Math. 31 (1975), no. 1, 17-41.

[Yau82] Stephen S. T. Yau, Various numerical invariants for isolated singularities, Amer. J. Math. 104 (1982), no. 5, 1063-1100.

Håkan Samuelsson Kalm, Martin Sera, Department of Mathematical Sciences, Division of Algebra and Geometry, University of Gothenburg and Chalmers University of Technology, SE-412 96 Göteborg, Sweden

E-mail address: hasam@chalmers.se

Martin Sera, Faculty of Engineering, Kyoto University of Advanced Science, Kyoto 615-8577, JAPAN

E-mail address: sera.martin@kuas.ac.jp 